\title{
Investigating the mass of the intermediate mass black hole candidate HLX-1 with the slimbh model
}

\author{
Odele Straub $^{1}$, Olivier Godet ${ }^{2,3}$, Natalie Webb ${ }^{2,3}$, Mathieu Servillat ${ }^{4,1}$, and Didier Barret ${ }^{2,3}$ \\ ${ }^{1}$ Laboratoire Univers et Théories, CNRS UMR 8102, Observatoire de Paris, Université Paris Diderot, 5 Place Jules Janssen, \\ 92195 Meudon, France \\ 2 Institut de Recherche en Astrophysique and Planétologie (IRAP), Université de Toulouse, UPS, 9 Avenue du colonel Roche, \\ 31028 Toulouse Cedex 4, France \\ e-mail: odele.straub@obspm.fr \\ 3 CNRS, UMR 5277, 31028 Toulouse, France \\ ${ }^{4}$ Laboratoire AIM, CEA Saclay, Bat. 709, 91191 Gif-sur-Yvette, France
}

Received 24 March 2014 / Accepted 14 July 2014

\section{ABSTRACT}

\begin{abstract}
In this paper we present a comprehensive study of the mass of the intermediate mass black hole candidate HLX-1 in the galaxy ESO 243-49. We analyse the continuum X-ray spectra collected by Swift, XMM-Newton, and Chandra with the slim disc model, slimbh, and estimate the black hole mass for the full range of inclination (inc $\left.=0^{\circ}-85^{\circ}\right)$ and $\operatorname{spin}\left(a_{*}=0-0.998\right)$. The relativistic slimbh model is particularly suited to study high luminosity disc spectra as it incorporates the effects of advection, such as the shift of the inner disc edge towards smaller radii and the increasing height of the disc photosphere (including relativistic ray-tracing from its proper location rather than the mid-plane of the disc). We find for increasing values of inclination that a zero spin black hole has a mass range of 6300-50900 $M_{\odot}$ and a maximally spinning black hole has a mass between 16900-191700 $M_{\odot}$. This is consistent with previous estimates and reinforces the idea that HLX-1 contains an intermediate mass black hole.
\end{abstract}

Key words. accretion, accretion disks - X-rays: binaries

\section{Introduction}

There is a limit to how luminous an object of a given mass can be. When a star or an accretion disc is in hydrostatic equilibrium it supports itself against gravity by its own internal radiation pressure. The critical luminosity (assuming isotropic emission) is thus given by the Eddington limit, $L_{\mathrm{Edd}}=4 \pi c G M / \kappa_{\mathrm{es}}=$ $1.26 \times 10^{38}\left(M / M_{\odot}\right) \mathrm{erg} / \mathrm{s}$, where $\mathrm{c}$ is the speed of light, $G$ is the gravitational constant, $\mathrm{M}$ is the mass of the gravitating body, $M_{\odot}$ is the solar mass, and $\kappa_{\mathrm{es}}=0.2(1+X) \mathrm{cm}^{2} / \mathrm{g}$ (with $X=1$ ) is the electron scattering opacity of a pure hydrogen plasma. There are, however, objects whose luminosities exceed this natural limit.

Ultraluminous X-ray sources (ULXs) are sources with X-ray luminosities $\gtrsim 10^{39} \mathrm{erg} / \mathrm{s}$. Most ULXs are thought to be powered by super-Eddington accretion onto a stellar mass black hole which can be accomplished (i) by powering strong disc winds (Shakura \& Sunyaev 1973; Lipunova 1999); (ii) by advecting the radiation along with the flow as in radiation pressure dominated disc models like Polish doughnuts (Abramowicz et al. 1978; Jaroszynski et al. 1980) and slim discs (Abramowicz et al. 1988); or (iii) both, advection and outflows (Poutanen et al. 2007; Dotan \& Shaviv 2011). Luminosities up to $10^{41} \mathrm{erg} / \mathrm{s}$ can therefore still be explained by super-Eddington mass accretion rates onto stellar mass black holes which can have maximum masses up to $\sim 80 M_{\odot}$. These higher mass black holes can be explained by direct collapse of metal poor stars (Belczynski et al. 2010).

The brightest known ULX in the sky is 2XMM J011028.1460421 in the lenticular galaxy ESO 243-49 $(z=0.0223$, Wiersema et al. 2010). With peak luminosities $\sim 10^{42} \mathrm{erg} / \mathrm{s}$ this object, dubbed HLX-1 (Farrell et al. 2009), belongs to a subclass of ULXs called the hyper-luminous X-ray sources (HLX, Gao et al. 2003). Like many X-ray binaries, HLX-1 shows transitions from low/hard to high/soft states
(Godet et al. 2009; Servillat et al. 2011), transient radio emission that can be associated with hard-to-soft transitions (Webb et al. 2012) and a weak optical counterpart (Soria et al. 2010). The extremely high luminosity of HLX-1 suggests, however, the presence of an intermediate mass black hole (IMBH) with a mass of about $100 M_{\odot}$ to $\sim 10^{5} M_{\odot}$.

Spectra of HLX-1 have already been studied with various disc models. These were either limited to one particular inclination and/or black hole spin, non- or semi-relativistic, or based on the standard Shakura \& Sunyaev (1973) disc and therefore only valid in the lowest luminosity regime, $L \lesssim 0.1 L_{\mathrm{Edd}}$. All previously used models agree that HLX-1 contains an IMBH. Servillat et al. (2011) predict a black hole mass $M>9000 M_{\odot}$ from fitting the non-relativistic diskbb model to multi-epoch data collected by Swift, XMM-Newton and Chandra. Davis et al. (2011) improve the mass constraints using a relativistic thin disc model with full radiative transfer, bhspec, and find $3000 M_{\odot}<M<3 \times 10^{5} M_{\odot}$. They assume a mass range of 1778-316228 $M_{\odot}$, consider spins $a_{*}=-1$ to 0.99 and luminosities between $0.03-1 L_{\text {Edd }}$ and fit simultaneously for the degenerate mass and spin parameters. As a consequence, a large fraction of their fits peg at the boundary value of at least one of these free parameters, and the results from different spectra are inconsistent with each other. In addition, most of their fits require luminosities far higher than the standard disc models allows. Godet et al. (2012) address the last point by employing a simplified slim disc model that includes Comptonisation and some relativistic corrections (Kawaguchi 2003) and estimate $M \sim 2 \times 10^{4} M_{\odot}$. They fit for a large mass range between $1-10^{5} M_{\odot}$ and have a disc structure that allows for high luminosities, but their study is limited to a face-on disc around a Schwarzschild black hole.

In this work we resolve the previous shortcomings regarding parameter space, luminosity regime and consistency among 
Table 1. Observational data.

\begin{tabular}{ccccc}
\hline \hline Obs. name & Instrument & Obs. ID & Start date & End date \\
\hline Swift & XRT & $00031287(001-252)$ & 2008-Oct.-24 & 2012-Sep.-16 \\
& & $00032577(001-011)$ & 2012-Oct.-02 & 2012-Nov.-11 \\
XMM-Newton & pn, MOS 1 and 2 & 0560180901 & 2008-Nov.-28 & 2008-Nov.-28 \\
Chandra & ACIS & 13122 & 2010-Sep.-06 & 2010-Sep.-07 \\
\hline
\end{tabular}

results from different spectra. We use a fully relativistic slim disc model (Sądowski et al. 2011; Straub et al. 2011) that accounts on the one hand for effects related to high mass accretion rates such as advection of radiation, relocation of the inner disc edge towards radii smaller than the innermost stable circular orbit (ISCO), and extended disc height. On the other hand, the model incorporates full vertical radiative transfer by integrating the emission of local annuli spectra and ray-tracing from the proper photosphere location. This slim disc model, slimbh, is valid for luminosities up to $1.25 L_{\mathrm{Edd}}$ and covers all inclinations and prograde spins (see the discussion of the model limits in Sect. 3). It differs from the well-known disc model bhspec, which is based on a standard thin disc, only in the structure of the underlying disc (the spectral differences between these two models have been studied in Straub et al. 2013, e.g. their Sect. 3.2 and the top panel in their Fig. 4). In comparison to the Kawaguchi (2003) slim disc, slimbh is fully relativistic and uses full radiative transfer.

We present results from fitting three thermal X-ray spectra of HLX-1 taken by Swift, XMM-Newton, and Chandra. With the employed slim disc model we are not only able to study the whole parameter plane spanned by black hole spin and inclination, we also get a consistent mass estimation for all spectra. This improves and solidifies the previous mass estimates. The paper contains a brief summary of the data selection in Sect. 2, a discussion about spectral fitting with the slim disc model in Sect. 3, and a summary of the results in Sect. 4. We finish with conclusions in Sect. 5

\section{X-ray data reduction}

The slim disc model is, like other $\alpha$-disc models, based on a few specific assumptions that limit its applicability to thermal state spectra. In particular it assumes that each plasma annulus is locally in thermal equilibrium and optically thick so that it radiates like a blackbody. In order to obtain data of the highest possible statistical quality showing a clear soft excess that we can interpret as emission coming from a multicolour blackbody disc, we make a few amendments to the data selection used in previous studies by Servillat et al. (2011), Davis et al. (2011), and Godet et al. (2012). We summed up the Swift data around the outburst peak to improve the statistics and employ the latest calibration files for the XMM-Newton data from 2008 (see below), but we omitted the observation from 2004 where the source was most likely in a steep power-law state (Davis et al. 2011; Godet et al. 2012). Only the Chandra spectrum remains the same. All data are listed in Table 1.

To enhance the visibility of a possible high energy tail the Swift spectrum consists of an accumulation of $\sim 121$ ks of Photon Counting (PC) data near the outburst peak between 2009 and 2012 (the plateau phase; see Godet et al. 2012). The SwiftXRT Photon Counting data (ObsID 31287 and 32577) were processed using the HEASOFT v6.14, the tool XRTPIPELINE v0.12.8 ${ }^{1}$,

\footnotetext{
1 http://heasarc.gsfc.nasa.gov/docs/swift/analysis/
}

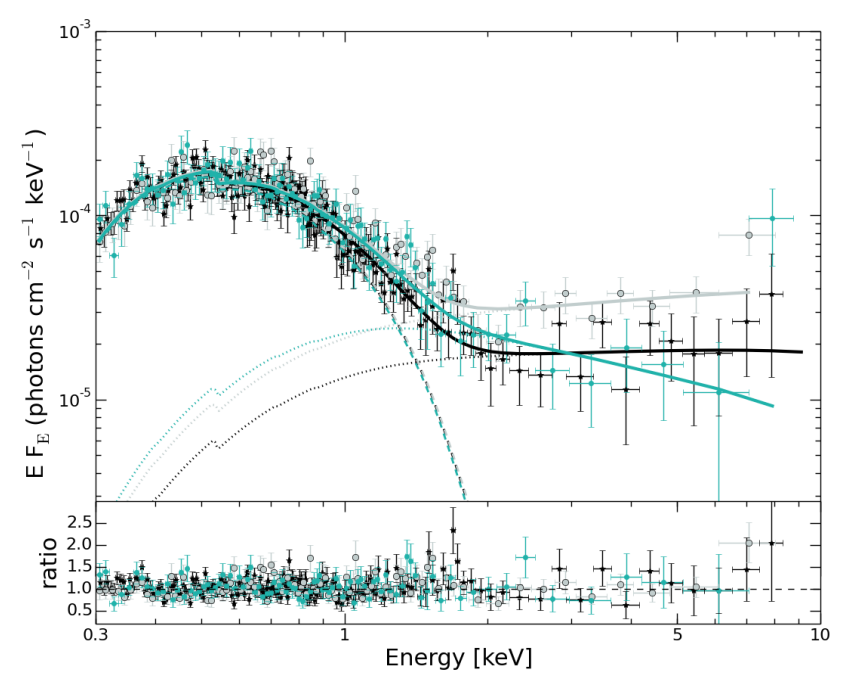

Fig. 1. The XMM-Newton pn (black) and MOS 1 and MOS 2 (cyan and grey) data. Fits were performed with slimbh for $a_{*}=0.5, i=60^{\circ}$ and $\alpha=0.01$. The full model (solid lines) is composed of an absorbed slim disc component (dashed lines) and a Compton component (dotted lines). See Table 2 for details.

and the calibration files (CALDB version 4.1). We used the grade 0-12 events, giving a slightly higher effective area at higher energies than the grade 0 events, and a 20 pixel (47.2 arcseconds) radius circle to extract the source and background spectra using XSELECT v2.4c. The background extraction region was chosen to be close to the source extraction region and in a region where we are sure that there are no sources present in the $X M M-N e w t o n$ field of view. The ancillary response files were created using XRTMKARF v0.6.0 and exposure maps generated by XRTEXPOMAP v0.2.7. We fit the spectrum using the response file SWXPC0TO12S6_20010101v012.RMF. Each spectrum was grouped to contain a minimum of 20 counts per bin to optimise the $\chi^{2}$ technique.

The XMM-Newton data were taken on 2008 November 28, whilst $H L X-1$ was still in the high/soft state. To reduce the XMM-Newton data we used the latest version of the $X M M-N e w t o n$ Science Analysis Software (SAS, version 13.5) and the latest calibration files (CCFs, May 2014). The MOS (Metal Oxide Semi-conductor) data were taken in full frame mode using the thin filter and the data were reduced using the emproc task of SAS. The event lists were filtered with the \#XMMEA_EM lag, and 0-12 of the predefined patterns (single, double, triple, and quadruple pixel events) were retained. The background was low and stable throughout the observation, resulting in $50.6 \mathrm{ks}$ of clean data. We also filtered in energy, using the range $0.2-10.0 \mathrm{keV}$. The pn-CCD data (from the positive-negative Charge Coupled Device camera) were taken in the small window mode and using the thin filter. The data were reduced using the SAS epproc task and 0-4 of the predefined patterns (single and double events) were retained, as 
these have the best energy calibration. Again the background was low and stable, resulting in $50.5 \mathrm{ks}$ of clean data. We used the \#XMMEA_EP filtering and the same energy range as for the MOS. We extracted the data using the optimised source extraction region. The MOS 1 spectra were extracted using circular regions with radii $36^{\prime \prime}$ and $43.5^{\prime \prime}$ for the source and background regions respectively. The background was chosen from a source free region close to the source. For the MOS 2 we used regions of $33^{\prime \prime}$ and $43.5^{\prime \prime}$ respectively and for the pn we used $24^{\prime \prime}$ and $28^{\prime \prime}$ for the source and background. We rebinned the data into $5 \mathrm{eV}$ bins as recommended in the SAS threads ${ }^{2}$. We used the SAS tasks rmfgen and arfgen to generate a redistribution matrix file and an ancillary response file, for each spectrum. The data were binned to contain at least 20 counts per bin.

\section{Modelling the thermal spectra of HLX-1}

The spectral model slimbh (after Abramowicz et al. 1988; Sadowski et al. 2011) is an additive disc model to be used in XSPEC (Arnaud 1996) and publicly available ${ }^{3}$. Like bhspec, this is a relativistic $\alpha$-disc model with full radiative transfer but instead of using the standard thin disc it is based on a slim disc, i.e. it incorporates the effects of advection. This means that with rising mass accretion rate an increasing fraction of photons gets trapped in the flow, carried inward, and is partly released at smaller radii. A typical slimbh disc is therefore softer at photon energies below the spectral peak and harder above it in comparison to bhspec.

The slimbh model has nine parameters: black hole mass $M$, black hole spin $a_{*}$, disc luminosity $L_{\text {disc }}$, inclination $i$, viscosity $\alpha$, distance $D$, hardening factor $f_{\text {hard }}$, limb darkening lflag and vertical extent $v$ flag. The latter two are flags to switch on/off the effect of limb darkening, and ray-tracing from the proper disc photosphere (both are switched on here). The hardening factor is calculated internally based on the TLUSTY grid of local annuli spectra (Hubeny \& Lanz 1995) which are then integrated over the whole disc (alternatively, $f_{\text {hard }}$ could also be set to a constant colour correction factor). Using the cosmological parameters from the WMAP5 results $\left(H_{0}=71 \mathrm{~km} \mathrm{~s}^{-1} \mathrm{Mpc}^{-1}\right.$, $\Omega_{\mathrm{M}}=0.27$ and $\Omega_{\Lambda}=0.73$ ), we adopt a source distance of $D=95 \mathrm{Mpc}$. The observed luminosity temperature relation in $\mathrm{X}$-ray binaries seems to favour low viscosities (Done \& Davis 2008); we therefore fix the viscosity parameter at $\alpha=0.01$. A discussion of how higher values of $\alpha$ affect the spectra can be found in Sect. 4.2. The inclination is fixed at values between the model limits $0^{\circ}-85^{\circ}$ in steps of $10^{\circ}$. The disc luminosity is left free to assume the best-fit value within the model limits $L_{\text {disc }}=0.05-1.25 L_{\text {Edd }}$. The TLUSTY grid calculates the specific intensity in a given direction from plane-parallel layers of radiation, whereas the surface of a slim disc becomes slanted with respect to the equator when the luminosity increases. Our current upper limits on the luminosity and inclination parameter are set by hand and represent the limits up to which we are confident that the internal calculation of the hardening factor is accurate.

As in the bhspec and kerrbb models the black hole mass and spin are also in slimbh degenerate parameters and cannot be fitted at the same time. When we leave both parameters free, the joint confidence contours for $M$ and $a_{*}$ tend to reveal either chains of local minima, a completely unconstrained $a_{*}$, or

\footnotetext{
2 http://xmm.esac.esa.int/sas/current/documentation/ threads/

3 http://astro.cas.cz/slimbh
}

the pegging of the spin and/or luminosity parameter at a boundary value. To perform better fits and avoid such inconclusive results either the mass or spin parameter must be fixed. We thus freeze the spin at values between $a_{*}=0.0-0.998$ in steps of 0.1 and fit for the mass. This procedure leads to well-constrained masses and luminosities that are consistent among the employed data sets. All three spectra show a notable amount of statistically low significance residuals below $2 \mathrm{keV}$ that might be due to narrow emission lines (see Godet et al. 2012). We do not try to fit these residuals and accept that the fit statistics for the XMM and Chandra spectra is slightly above the optimum.

The Swift and XMM-Newton data were fitted with the same model, tbabs $\times($ slimbh + nthc $)$ and the Chandra data were in addition multiplied by pileup (see Servillat et al. 2011). The pn, MOS 1, and MOS 2 data sets were fitted jointly with a tied mass parameter. The absorption component, tbabs, is used to account for the total neutral hydrogen column density along the observer's line of sight, $N_{\mathrm{H}}=4 \times 10^{20} \mathrm{~cm}^{-2}$ (Farrell et al. 2009; Godet et al. 2012). The thermal Compton component, nthc, consists of the free power-law photon index $\Gamma$, the electron temperature $k T_{\mathrm{e}}$, the seed photon temperature $k T_{\mathrm{bb}}=0.2 \mathrm{keV}$ (Servillat et al. 2011; Godet et al. 2012), the input type for discblackbody seed photons, the redshift $z=0.0223$ (Wiersema et al. 2010) and the normalisation (free). Because of the relatively large uncertainty in the data points at the highest energies the fits are not sensitive to the electron temperature. Its precise value neither influences the fitting parameters nor the goodness of fit. We therefore fix $k T_{\mathrm{e}}=5 \mathrm{keV}$ at an arbitrary value. The pileup component uses a frame time of $0.83 \mathrm{~s}$ and has only one free (grade morphing) parameter that can assume values between 0 and 1. Figure 2 and Table 2 show the results of a fit with black hole spin $a_{*}=0.5$ and inclination $i=60^{\circ}$.

\section{The mass of HLX-1}

We study the parameter plane spanned by black hole spin and inclination and find the distribution of black hole mass shown on the left panel in Fig. 3. The shaded areas indicate the $90 \%$ confidence interval for the whole spin range. Singled out as black lines are the masses (90\% confidence) we obtained for $a_{*}=0.5$. The open and filled circles mark previous results (error bars have been omitted) of Davis et al. (2011) and Godet et al. (2012), respectively. The mass estimates from the Chandra spectrum (grey), which is affected by pileup, seem to slightly overestimate the black hole mass as compared to the Swift (green) and XMM (blue) spectra which are almost congruent. They are, however, consistent within the error bars. This behaviour does not apply to the results of Davis et al. (2011), where the highest mass at a given inclination is associated with a Swift or XMM spectrum and a spin that pegs at the maximum value. In general, higher inclinations implicate larger black hole masses as the geometrically induced reduced photon count is compensated with the larger emitting area of a higher source mass. With increasing spin the radiation originates from an ever smaller area around the black hole, which also translates into a reduced amount of photons and consequently a larger black hole mass. To show the effect of the black hole spin in detail we plot in the right panel of Fig. 3 the masses for Swift spectrum alone. Given a Schwarzschild black hole and a face-on disc, the mass found by Godet et al. (2012) is substantially higher than the one found with models that include full radiative transfer.

The XMM spectrum requires a significant amount of Comptonisation to model the high energy tail (see Fig. 2). The contribution of the disc to the total luminosity is thus 


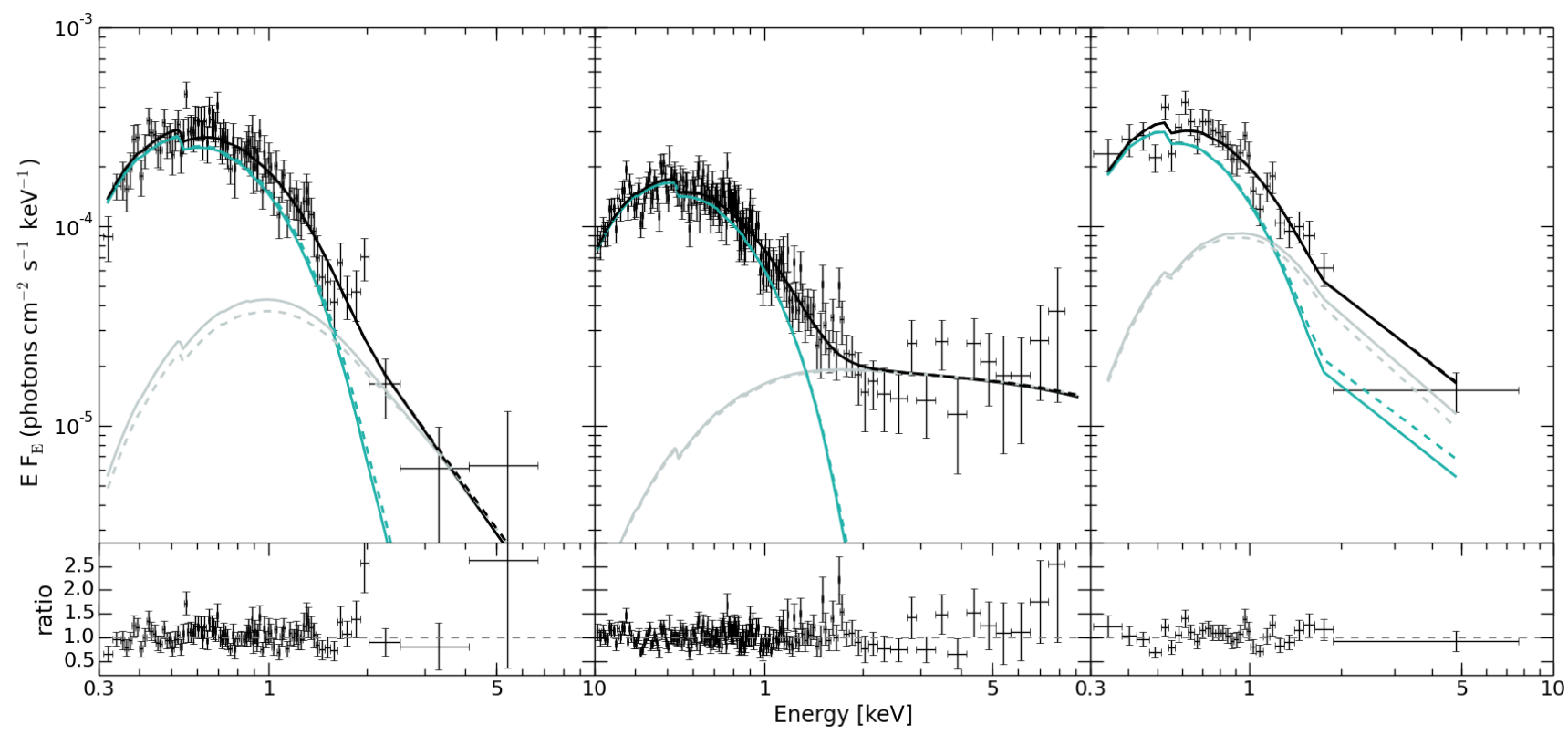

Fig. 2. Swift (left), XMM-Newton pn (middle) and Chandra (right) spectra fitted with slimbh. Each model consists of a disc and a Compton component (cyan and grey, respectively) and represents the best fit for the case $a_{*}=0.5, i=60^{\circ}$ and $\alpha=0.01$ (compare Table 2). The dashed components indicate the best fits of the same configuration when $\alpha=0.1$.

Table 2. Detailed results for Swift, XMM-Newton and Chandra spectra for $a_{*}=0.5$ and $i=60^{\circ}$.

\begin{tabular}{|c|c|c|c|}
\hline Model parameter & Swift & XMM-Newton (pn, MOS 1, MOS 2) & Chandra \\
\hline tbabs & \\
\hline$N_{\mathrm{H}}\left[\times 10^{20}\right]$ & & 4 & \\
\hline slimbh & \multirow{3}{*}{$19989_{-3078}^{+5883+13902}-4606$} & & \multirow{3}{*}{$29952_{-8381-12201}^{+14476+36285}$} \\
\hline$M\left[M_{\odot}\right]$ & & $18473_{-1074-1905}^{+1195+2323}$ & \\
\hline$a_{*}$ & & 0.5 & \\
\hline$L_{\mathrm{disc}}\left[L_{\mathrm{Edd}}\right]$ & \multirow{3}{*}{$0.64_{-0.19}^{+0.14}$} & $0.43_{-0.02}^{+0.02}, 0.43_{-0.03}^{+0.03}, 0.42_{-0.03}^{+0.03}$ & \multirow[t]{3}{*}{$0.62_{-0.18}^{+0.25}$} \\
\hline$i[\mathrm{deg}]$ & & 60 & \\
\hline$\alpha$ & & 0.01 & \\
\hline$D[\mathrm{Mpc}]$ & \multirow{5}{*}{$4.16_{-2.57}^{+3.24}$} & 95 & \multirow{5}{*}{$4.55_{-2.50}^{+2.58}$} \\
\hline nthc & & & \\
\hline$\Gamma$ & & $1.94_{-0.25}^{+0.30}, 1.84_{-0.21}^{+0.24}, 2.49_{-0.43}^{+0.53}$ & \\
\hline$k T_{\mathrm{e}}[\mathrm{keV}]$ & & 5 & \\
\hline$k T_{\mathrm{bb}}[\mathrm{keV}]$ & & 0.2 & \\
\hline$z$ & \multirow{3}{*}{$4.59_{-4.23}^{+7.27}$} & 0.0223 & \multirow{3}{*}{$11.96_{-10.36}^{+9.12}$} \\
\hline$N\left[10^{-5}\right]$ & & $1.45_{-0.37}^{+0.45}, 2.38_{-0.53}^{+0.61}, 2.52_{-0.76}^{+0.90}$ & \\
\hline pileup & & & \\
\hline$t_{\text {frame }}[\mathrm{s}]$ & - & - & 0.83 \\
\hline$f$ & - & - & $0.16_{-0.16}^{+0.61}$ \\
\hline$F_{\text {disc }}\left[10^{-13} \mathrm{erg} / \mathrm{cm}^{2} / \mathrm{s}\right]$ & 6.867 & 3.806 & 9.088 \\
\hline$\chi^{2} /$ d.o.f. $\left(\chi_{r}^{2}\right)$ & $82.07 / 85(0.97)$ & $367.34 / 361(1.02)$ & $37.84 / 27(1.40)$ \\
\hline
\end{tabular}

Notes. The two error intervals for the black hole mass corresponds to the $90 \%$ and $3 \sigma$ confidence level, respectively. The unabsorbed disc flux, $F_{\text {disc }}$, is taken over the energy range $0.3-10 \mathrm{keV}$.

substantially lower than for the Swift and Chandra spectrum, as shown in the left panel of Fig. 4. Both increasing spin and increasing inclination reduce the effective emission area which is efficiently compensated with increasing black hole masses during the fit procedure, while the disc luminosity does not change significantly. If, however, the configuration is set to maximum spin, the model spectrum becomes so soft below the peak that tuning the mass parameter alone is not sufficient anymore to fit the observed spectrum, instead the disc luminosity needs to be increased. This behaviour is visible in the right panel of Fig. 4, where the luminosity that corresponds to the maximum spin crosses all other lines. In practice this means that in a low inclination system with uncertain black hole mass a low spin could be easily mistaken for maximally rotating black hole.

There is currently no evidence of eclipses in HLX-1, the inclination is thus likely to be lower than about $75^{\circ}$. We assume here an inclination of $60^{\circ}$ to demonstrate the modelling of the three spectra for a moderate spin, $a_{*}=0.5$, in Fig. 2 and the 

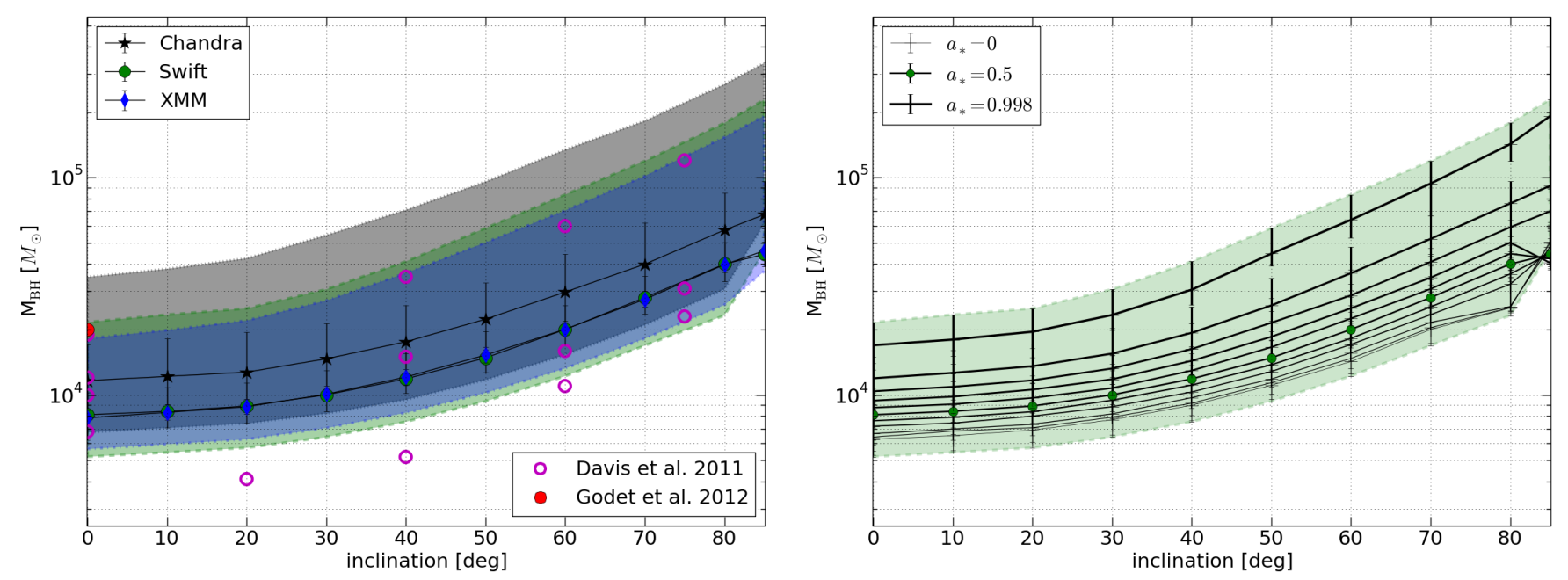

Fig. 3. The black hole mass in HLX-1. Left: the shaded areas indicate the 90\% confidence interval for the mass estimation of the Chandra (grey), Swift (green), and XMM (blue) spectrum, respectively, given for the whole spin range. The three solid lines represent the black hole mass that corresponds to the spin $a_{*}=0.5$ (where the error bars denote $90 \%$ confidence). The filled and open circles show previous mass measurements (see text for details). Right: the influence of spin on the mass estimation is shown for the Swift data. Error bars give the $90 \%$ confidence interval.
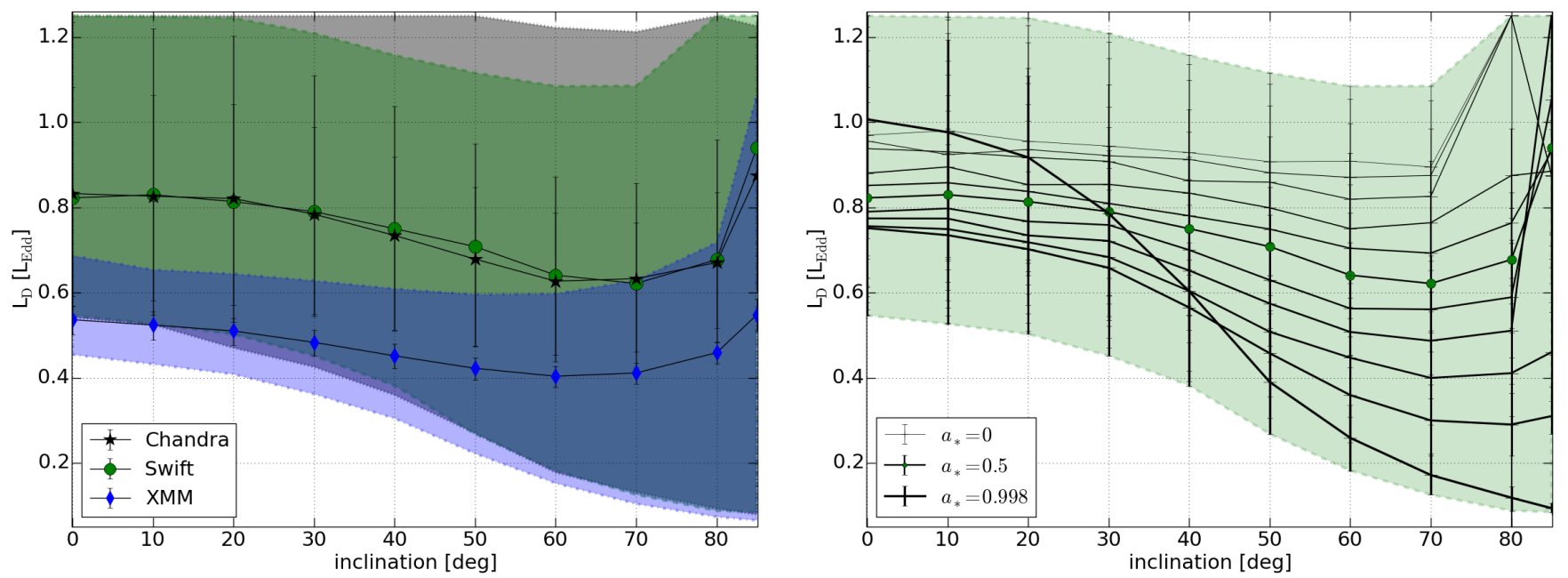

Fig. 4. The disc luminosity in HLX-1. Left: the shaded areas indicate the $90 \%$ confidence interval for the luminosity distribution associated with the black hole mass measured in Fig. 3. Again, the the result for $a_{*}=0.5$ is singled out and given by the three solid lines (with $90 \%$ confidence), corresponding, respectively, to the Chandra (grey), Swift (green) and XMM (blue) spectrum. Right: the influence of the black hole spin on the luminosity of the Swift spectrum. Error bars give the $90 \%$ confidence interval.

resulting parameter values in Table 2. Because of the weakness of the high energy tail in the Swift and Chandra spectra the photon index, $\Gamma$, is only weakly constrained. Moreover, in the chosen example the adding of a Compton component is statistically not very significant, in particular for the Chandra spectrum; the F-test in XSPEC gives a probability of $p=3 \times 10^{-18}, p=0.008$, and $p=0.178$ for the XMM, Swift, and the Chandra data, respectively. However, as we move through the parameter space to lower black hole spins and inclinations, the Compton component becomes more significant, even for the Swift and Chandra observation. This is because for low spin and low inclination pure absorbed disc models are intrinsically too soft to properly reproduce the high energy $(>4 \mathrm{keV})$ photons. As a consequence, the models with the lowest spins and inclinations, $a_{*} \lesssim 0.1$ and $i \lesssim 20^{\circ}$, respectively, favour high disc luminosities; without the Compton component they would require a luminosity higher than our current limit of $L=1.25 L_{\mathrm{Edd}}$. Whether such models would ultimately fit the data better remains to be seen in future work.

\subsection{The mass accretion rate}

The efficiency of an accretion disc, $\eta=1-u_{t}\left(r_{\text {in }}\right) / c^{2}$, defines how much of the accreted mass can be converted into luminosity,

$L=\eta \dot{M} c^{2}$.

The relevant quantity is the specific energy at the inner disc edge, $u_{t}\left(r_{\text {in }}\right)$. While the inner disc radius in standard discs is given by the ISCO, so that the standard disc efficiency, $\eta_{*}$, only depends on the black hole spin, in slim discs there is an additional dependency on the mass accretion rate that moves the slim 


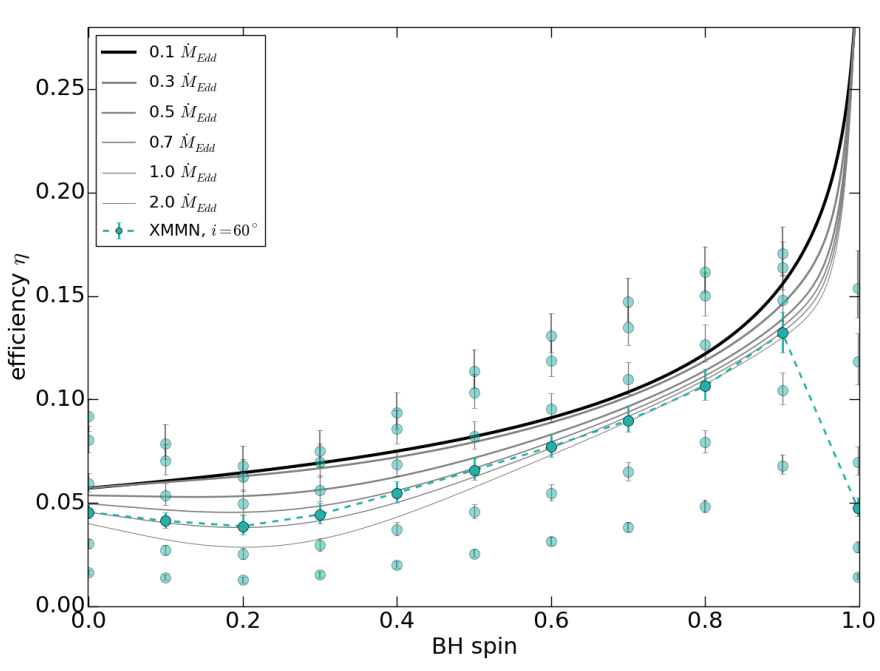

Fig. 5. Slim disc efficiency. The solid lines indicate model efficiencies for a number of mass accretion rates. The black line corresponds to the standard thin disc efficiency. For HLX-1 we derive effective efficiencies from the fitted disc luminosity of the XMM-Newton spectrum and the observed unabsorbed $0.3-10 \mathrm{keV}$ disc luminosity for selected inclinations $\left(i=0^{\circ}, 30^{\circ}, 50^{\circ}, 60^{\circ}, 70^{\circ}\right.$, and $\left.80^{\circ}\right)$. The dashed line highlights the spin evolution of the effective efficiency for a disc with $i=60^{\circ}$. Error bars include the uncertainty in black hole mass and disc luminosity.

edge from the ISCO towards the marginally bound radius, $r_{\mathrm{mb}}$. This means the slim disc efficiency decreases for increasing mass accretion rates and formally goes to zero because $u_{t}\left(r_{\mathrm{mb}}\right)=1$. In Fig. 5 we show the behaviour of the standard and slim disc efficiency with black hole spin. To obtain the mass accretion rate from the fitted model luminosity we first rewrite Eq. (1) using the normalised mass accretion rate $\dot{m} \equiv \dot{M} / \dot{M}_{\text {Edd }}$ and $\dot{M}_{\text {Edd }} \equiv L_{\text {Edd }} /\left(\eta_{*} c^{2}\right)$ for the Eddington limit on the mass accretion rate ${ }^{4}$. Then we employ the approximate formula of the potential spout inner edge given in Abramowicz et al. (2010),

$$
\begin{aligned}
r_{\text {in }}= & \operatorname{Min}\left[\left(0.275-0.410 a_{*}+0.143 a_{*}^{2}\right) \dot{m}^{-1.4}\right. \\
& \left.+4.454 .87 a_{*}+8.06 a_{*}^{2}-6.38 a_{*}^{3} 0.985 r_{\text {ISCO }}\right]
\end{aligned}
$$

to find the inner radius of the slim disc and obtain an expression for $\eta$ that depends only on $a_{*}$ and $\dot{m}$. From the slim disc fits one obtains the modelled disc luminosity, so that Eq. (1) finally can be solved for $\dot{m}$. This results in mass accretion rates between $\dot{M} \simeq 7 \times 10^{-5}$ and $2 \times 10^{-3} M_{\odot} / y$. The highest mass accretion rates are achieved for slowly rotating black holes with highly inclined accretion discs. This agrees well with the results of Godet et al. (2012) who calculated the mass accretion rate during the outbursts to be $\sim 1.2 \times 10^{-4} M_{\odot} / \mathrm{y}$ and $\sim 8.2 \times 10^{-5} M_{\odot} / \mathrm{y}$ assuming a Schwarzschild black hole and a face-on disc. The corresponding model efficiency takes values between $\eta \simeq(0.045-0.049) \pm 0.001$ for a Schwarzschild black hole (with $i=85^{\circ}-0^{\circ}$ ) and $\eta \simeq 0.32$ for a maximally rotating Kerr black hole (independent of inclination). Next, we look at the observed bolometric disc luminosity, $L_{\mathrm{bol}}=4 \pi D^{2} F_{\mathrm{obs}}$, calculated from the $0.3-10 \mathrm{keV}$ unabsorbed disc flux. One can use Eq. (1) to compare the observed luminosity output to the fitted mass accretion rate and obtain the effective efficiency of the accretion disc in HLX-1. We show the results in Fig. 5. As Godet et al. (2012) state, a Schwarzschild black hole with a face-on disc is relatively efficient with $\eta \simeq 0.1$. This also holds

\footnotetext{
4 Another common definition is $\dot{M}_{\text {Edd }}=L_{\text {Edd }} / c^{2}$.
}

for low inclinations, $i<30^{\circ}$, and moderate black hole spins, $0.4<a *<0.9$. We find that starting from an inclination of $30^{\circ}$ the disc becomes increasingly inefficient, in particular for minimal and maximal spins. Above an inclination of $\sim 50^{\circ}$ the disc has become an entirely inefficient emitter, i.e. the effective efficiency is for all spins significantly lower compared to a standard disc. This means on the one hand that the best fitting models to inclined discs require substantial mass accretion rates that can only be matched to the comparatively low luminosity output if the disc radiates inefficiently. On the other hand, if we see the disc nearly face-on, even a small accretion rate may generate a huge luminosity. To determine whether the disc in HLX-1 is advection dominated or over-efficient, better constraints on the inclination are required.

\subsection{The effect of viscosity}

In hydrodynamic disc models like bhspec and slimbh the relation between viscous stresses and pressure is parametrised by the viscosity parameter, $\alpha$. Although accretion discs have been studied for forty years, the precise nature of this relation is still unclear and $\alpha$ remains a phenomenological quantity that possibly comprises several physical mechanisms related to the pressure balance in accretion discs. We use a default value of $\alpha=0.01$ which is supported by observations of high luminosity, radiation pressure dominated discs that require a roughly constant colour temperature correction (i.e. hardening factor) which is only supported by a low viscosity (Done \& Davis 2008). We note, however, that outburst cycles in low mass X-ray binaries require a very efficient angular momentum transport and thus suggest a high $\alpha$ value (see e.g. the review by Lasota 2001). We therefore perform an additional analysis assuming $\alpha=0.1$ and find that the black hole mass increases by $7-14 \%$. Figure 2 shows the corresponding best fit as dashed lines. Since a larger viscosity translates into a smaller amount of thermalised photons in the plasma, which in turn entails a larger colour temperature correction, the slim disc spectra of a given luminosity get harder with increasing $\alpha$. This change in the spectral shape causes a roughly $10 \%$ increase in mass. We caution, however, that a high luminosity disc becomes effectively optically thin when the viscosity is too large and therefore consider the low viscosity value as the standard (see also the discussion in Straub et al. 2011, 2013).

\section{Conclusions}

We analysed three spectra of the IMBH candidate HLX-1 that were collected by Swift, XMM-Newton, and Chandra during different missions between 2008 and 2012. We estimate the black hole mass using the fully relativistic slim disc model, slimbh (Sagdowski et al. 2011; Straub et al. 2011), which allows us to self-consistently probe the trans-Eddington luminosity regime in the whole parameter plane spanned by black hole spin and inclination. This addresses and remedies the deficits of previously used models which were either not relativistic (Servillat et al. 2011), only valid at lowest luminosities (Davis et al. 2011), or only valid for one particular inclination and spin (Godet et al. 2012). Assuming a low disc viscosity $(\alpha=0.01)$ we find that a Schwarzschild black hole has a mass of about 6300-50900 $M_{\odot}$ (increasing with disc inclination), whereas a maximally spinning black hole has a mass between 16900-191700 $M_{\odot}$. A high viscosity disc $(\alpha=0.1)$ has black hole masses that are roughly $10 \%$ higher. This result is consistent among all three observations with Swift, XMM-Newton, and Chandra. Moreover, it is also in good agreement with earlier measurements based on 
bhspec (Davis et al. 2011) and other slim disc models (Godet et al. 2012).

The continuum fitting method that we have applied here determines the inner edge of the accretion disc given its effective temperature and flux, i.e. it is designed to measure the black hole spin and relies strongly on the knowledge of the binary parameters, $M, D$, and $i$. Given that the inclination is only constrained to $i<75^{\circ}$ the constraints on the black hole mass are necessarily fairly weak. Nonetheless, our results clearly place HLX-1 in the regime of IMBHs. Future dynamical measurements of the binary parameters of HLX-1 will allow us to apply the continuum fitting method as it has been intended, namely to assess the spin of the IMBH.

Acknowledgements. We thank S. Farrell for his invaluable feedback on the original manuscript and the anonymous referee for their helpful remarks. O.S. thanks in particular M. Bursa for the continuous and illuminating discussions about slim discs.

\section{References}

Abramowicz, M., Jaroszynski, M., \& Sikora, M. 1978, A\&A, 63, 221 Abramowicz, M. A., Czerny, B., Lasota, J. P., \& Szuszkiewicz, E. 1988, ApJ, 332,646
Abramowicz, M. A., Jaroszyński, M., Kato, S., et al. 2010, A\&A, 521, A15 Arnaud, K. A. 1996, in Astronomical Data Analysis Software and Systems V, eds. G. H. Jacoby \& J. Barnes, ASP Conf. Ser., 101, 17

Belczynski, K., Bulik, T., Fryer, C. L., et al. 2010, ApJ, 714, 1217

Davis, S. W., Narayan, R., Zhu, Y., et al. 2011, ApJ, 734, 111

Done, C., \& Davis, S. W. 2008, ApJ, 683, 389

Dotan, C., \& Shaviv, N. J. 2011, MNRAS, 413, 1623

Farrell, S. A., Webb, N. A., Barret, D., Godet, O., \& Rodrigues, J. M. 2009, Nature, 460, 73

Gao, Y., Wang, Q. D., Appleton, P. N., \& Lucas, R. A. 2003, ApJ, 596, L171

Godet, O., Barret, D., Webb, N. A., Farrell, S. A., \& Gehrels, N. 2009, ApJ, 705, L109

Godet, O., Plazolles, B., Kawaguchi, T., et al. 2012, ApJ, 752, 34

Hubeny, I., \& Lanz, T. 1995, ApJ, 439, 875

Jaroszynski, M., Abramowicz, M. A., \& Paczynski, B. 1980, Acta Astron., 30, 1 Kawaguchi, T. 2003, ApJ, 593, 69

Lasota, J.-P. 2001, New Astron. Rev., 45, 449

Lipunova, G. V. 1999, Astron. Lett., 25, 508

Poutanen, J., Lipunova, G., Fabrika, S., Butkevich, A. G., \& Abolmasov, P. 2007, MNRAS, 377, 1187

Sa̧dowski, A., Abramowicz, M., Bursa, M., et al. 2011, A\&A, 527, A17

Servillat, M., Farrell, S. A., Lin, D., et al. 2011, ApJ, 743, 6

Shakura, N. I., \& Sunyaev, R. A. 1973, A\&A, 24, 337

Soria, R., Hau, G. K. T., Graham, A. W., et al. 2010, MNRAS, 405, 870

Straub, O., Bursa, M., Sądowski, A., et al. 2011, A\&A, 533, A67

Straub, O., Done, C., \& Middleton, M. 2013, A\&A, 553, A61

Webb, N., Cseh, D., Lenc, E., et al. 2012, Science, 337, 554

Wiersema, K., Farrell, S. A., Webb, N. A., et al. 2010, ApJ, 721, L102 